\title{
Considerações sobre as relações entre a análise de citação e a pesquisa científica colaborativa
}

\author{
Considerations on the relationships between scientific \\ collaborative research and citation analysis
}

\author{
Márcia de Oliveira TEIXEIRA' \\ Carlos José Saldanha MACHADO² \\ Ana Tereza Pinto FILIPECKI ${ }^{3}$ \\ Lia HASENCLEVER ${ }^{4}$ \\ Helena Espellet KLEIN 5
}

\section{RESUMO}

Agências de fomento governamentais e organismos internacionais estimulam programas de pesquisas científicas colaborativas. E um dos principais argumentos são os seus benefícios para o aumento da produtividade científica. Segundo a literatura especializada, as colaborações científicas, ao fortalecerem as dimensões multi e interdisciplinar, potencializam o incremento da produção de inovações técnico-científicas em diferentes setores. Assim se deu o crescimento do interesse nos indicadores de produtividade das colaborações, sendo os mais difundidos apoiados na análise da citação. Todavia até que ponto as medições baseadas nessa análise nos permitem dimensionar os efeitos das colaborações científicas na produção e no conteúdo de conhecimentos científicos? O objetivo deste trabalho é discutir os limites da análise da citação para a avaliação de iniciativas de pesquisa científica colaborativa, a partir da revisão de proposições da literatura especializada.

Palavras-chave: pesquisa colaborativa; pesquisa científica; produtividade; cienciometria; estatísticas da ciência.

\section{ABSTRACT}

Governmental funding agencies and international non-governmental organizations promote collaborative research programs. One of their main arguments is the benefits for increasing scientific productivity. According to

1 Pesquisadora do Laboratório de Iniciação Científica-Programa de Vocação Científica / Escola Politécnica de Saúde Joaquim Venâncio / Fundação Oswaldo Cruz. Endereço para correspondência: Av. Brasil, 4365, Manguinhos, Rio de Janeiro, RJ, Brasil. Correpondência para/Correspondence to: M.O. TEIXEIRA. E-mail: <marciat@fiocruz.br>.

2 Pesquisador do Laboratório de Pesquisa em Ciência, Tecnologia e Inovação em Saúde / Instituto de Comunicação e Informação Científica e Tecnológica em Saúde / Fundação Oswaldo Cruz. Email: <csaldanha@cict.fiocruz.br>.

3 Pesquisadora do Laboratório de Iniciação Científica-Programa de Vocação Científica / Escola Politécnica de Saúde Joaquim Venâncio / Fundação Oswaldo Cruz. E-mail: <afilipecki@fiocruz.br>.

4 Pesquisadora e Professora Adjunta do Instituto de Economia, UFRJ. E-mail: <lia@ie.ufri.br>.

${ }^{5}$ Mestranda em Políticas Públicas, Estratégias e Desenvolvimento da Universidade Federal do Rio de Janeiro, Instituto de Economia, UFRJ.E-mail: <helenaklein@gmail.com>

Recebido em 22/4/2009 e aceito para publicação em 20/8/2009. 
specialized literature, when scientific collaborations support the multi-disciplinary dimensions, they promote the development of techno-scientific production in different fields of action. In this way, the growing interest in productivity indicators of scientific collaborations was unfolded and the indicators based on citation analysis are the best known ones. Nevertheless, how much of the measures based on this kind of analysis can reveal the effects of scientific collaboration on the production and content of scientific knowledge? So, the aim of this paper is to discuss the limits of citation analysis to evaluate initiatives of collaborative scientific research based on the propositions of specialized literature.

Keywords: collaborative research; scientific research; productivity; scientometry; science statistics.

\section{INTRODUÇÃO}

Nas últimas décadas, agências de fomento governamentais e organismos internacionais estimularam o desenvolvimento de programas de pesquisa científica colaborativa entre laboratórios ou entre instituições de pesquisa (Bozerman; Boardman, 2003; Chompalov; Shrum, 1999; Katz; Martin, 1997; Longo; Oliveira, 2000; Shrum; Chompalov, 2007). A literatura especializada na análise da pesquisa científica colaborativa identifica diferentes fatores associados a esse estímulo. Os mais recorrentes são o volume de projetos que necessitam de um maior aporte de recursos, o recrutamento de pesquisadores com expertise em diferentes áreas do conhecimento, além da manipulação de instrumentações complexas e diversificadas, incorporadas ou não a equipamentos (Beaver, 2001; Chompalov; Shrum, 1999; Katz; Martin, 1995, 1997; Larsen, 2008; Shrum; Shrum; Chompalov, 2007; Teixeira et al., 2009a, 2008c).

Um dos principais argumentos para a disseminação dessa prática, porém, são os seus benefícios para elevar a produtividade da pesquisa, em especial de artigos científicos em publicações de alto impacto (Beaver, 2001; Bozerman; Boardman, 2003; Larsen; 2008; Wray; 2006). Segundo a literatura especializada, o aumento da produtividade também é um dos fatores associados ao incremento da produção de inovações técnico-científicas em diferentes setores da economia (Cummings; Kiesler, 2005; Longo; Oliveira, 2000; Sonnenwald; 2003), mormente em novas áreas do conhecimento como a nanobiotecnologia (Larsen, 2008). Assim, simultaneamente àquele processo, ocorreu o crescimento do interesse de organismos e de especialistas no desenvolvimento e no aperfeiçoamento de indicadores capazes de medirem a produtividade das pesquisas colaborativas. Os indicadores mais difundidos baseiam-se nos pressupostos e nas ferramentas da cienciometria (Leydesdorff; 2001) e utilizam como foco principal para suas medições a análise da citação, bem como a vinculação institucional de autores e coautores (Beaver, 1984; Katz; Martin, 1995, 1997; Knoben; Oerlemans, 2006). O foco na vinculação está diretamente relacionada a uma característica da pesquisa científica colaborativa, qual seja, a dispersão de colaborações entre diferentes instituições, formando colaborações multi-institucionais entre duas ou mais instituições (Chompalov; Shrum, 1999; Cummings; Kiesler, 2005; Shrum; Chompalov, 2007).

Todavia, quando analisamos as métricas usualmente empregadas para o estudo da produtividade em pesquisas científicas colaborativas, algumas questões se apresentam. Até que ponto a métrica baseada nessa análise nos permite dimensionar os efeitos das colaborações científicas na produção de conhecimentos científicos? A análise de citações é uma medida do conhecimento técnico-científico produzido por uma colaboração científica? A presença de múltiplos autores caracteriza de fato uma pesquisa científica desenvolvida no âmbito da colaboração entre equipes de diferentes laboratórios ou instituições? Nesse sentido, essa métrica permite avaliar o impacto dos programas colaborativos na produção de conhecimentos técnicocientíficos com potencial inovador para os diferentes setores da economia?

A partir desse conjunto de questões, o objetivo deste artigo é identificar e discutir os limites da métrica de citação para o estudo e a avaliação de iniciativas de pesquisa científica colaborativa. $\bigcirc$ artigo está dividido em quatro seções. Iniciamos com uma nota metodológica. Em seguida, brevíssimas considerações 
sobre a disseminação da métrica da ciência e tecnologia (C\&T) e sobre a cienciometria. A terceira seção é dedicada à discussão da correlação entre o aumento de publicações com múltiplos autores, a possibilidade de utilizar esse fenômeno como métrica da produção de conhecimentos científicos em pesquisas colaborativas e a dinâmica da pesquisa científica colaborativa. Por fim, indicamos alguns pontos para a discussão de novos mecanismos para a avaliação de iniciativas de pesquisa científica colaborativa na América Latina e no Caribe (Velho, 1999).

\section{Nota metodológica}

Este artigo é fruto de uma série de discussões encetadas pelos autores durante a revisão bibliográfica da literatura especializada na análise da pesquisa científica colaborativa. Tal revisão foi suscitada pelo desenvolvimento de um estudo de caso sobre o uso de redes cooperativas como dispositivo de coordenação de programas de indução à pesquisa técnico-científica colaborativa (Teixeira et al., 2008a,2008c; 2009a; 2009 b; 2009c). Nosso foco são os limites das ferramentas usualmente empregadas para analisar os efeitos da pesquisa científica colaborativa na produtividade, construído a partir das nossas tentativas de usar essas ferramentas para analisar o material empírico do nosso próprio estudo de caso.

Partimos das reflexões de Godin $(2005 ; 2008)$ para tratar da métrica da ciência e da tecnologia (C\&T) e sua história, bem como das contribuições de Polanco $(1995 ;$ 2008) sobre a cienciometria. Encontramos no trabalho de Katz e Martin (1995, 1997) uma importante introdução ao debate da métrica da produtividade em pesquisas científicas colaborativas. Destacamos também as contribuições de Subramanyam (1983) sobre estudos bibliométricos. Para a análise da dinâmica da colaboração e seus efeitos para a divisão de trabalho e conformação de equipes, fundamental para a compreensão dos limites da métrica baseada na análise de citação, lançamos mão dos trabalhos de Sonnenwald (2003), Hara et al. (2003), além das revisões realizadas por San Martín-Rodriguez et al. (2005) e D'Amour et al. (2005).

\section{Métricas, Cienciometria e Análise de citação - Antecedentes}

A disseminação da análise de citação como métrica da pesquisa científica colaborativa faz parte da generalização dos esforços de mensuração da ciência e da tecnologia. $\bigcirc$ interesse de diferentes atores como pesquisadores e gestores de organismos internacionais pela medida das ciências não é exatamente recente. De fato, os primeiros esforços remontam ao final do século XIX, como relata Godin $(2005 ;$ 2008), muito embora não possamos traçar uma linha singela de continuidade entre esses esforços. Há diferenças marcantes entre as concepções de ciência e tecnologia que orientam a produção de medida, o modo de medir e, sobretudo, quem mede (Godin, 2005; 2008; Teixeira, 2008c). A partir da década de 1940, a realização de medidas tornou-se uma atividade para especialistas alojados em instituições governamentais e organismos internacionais, os quais passaram a estabelecer metodologias para as medidas a exemplo do Manual de Frascati (OCDE, 1962) elaborado pela Organização para a Cooperação e o Desenvolvimento Econômico (OCDE).

Consideramos, para os objetivos deste artigo, sempre baseados nas proposições de Godin (2005; 2008), que: a) as métricas ou estatísticas de ciência e tecnologia têm história, assim como as ciências que elas devem medir; b) as estatísticas possuem objetivos precisos e prévios ao exercício de medir, quais sejam, determinar os alvos (temas e áreas) o recebimento de investimentos, bem como o nível desses investimentos.

A cienciometria, por seu turno, é uma métrica específica de publicações, de autores e de citações, a qual considera artigos científicos como indicadores da produção da pesquisa científica (Polanco; 1995; Velho; 1999). Nesse sentido, e baseada nas concepções de Price e Beaver (1966), a cienciometria concebe a ciência a partir da literatura científica (Leydesdorff; 2001).

Quanto aos métodos, a cienciometria utiliza essencialmente os quantitativos para reunir e tratar um volume expressivo de dados calculados a partir da análise estatística de publicações científicas. Portanto ela é intensiva no uso de ferramentas de tecnologias de informação. Os cienciometristas dispõem, por conseguinte, de uma variedade de sistemas para a análise da informação técnico-científica armazenada em bases de dados bibliográficas, como a mantida pelo Information Science Institute (ISI). A tecnologia da informação também permite o cruzamento de dados extraídos de diferentes bases e o cruzamento de diferentes variáveis - instituição, período, área de conhecimento, tema de pesquisa (Polanco, 2006). Dado o número de 
artigos publicados e de periódicos em circulação, a principal vantagem dos sistemas de tecnologia da informação é a possibilidade de suportarem o tratamento de um grande volume de dados (Polanco, 2006, 2008; Leydesdorff, 2001). Os sistemas tornam possível a identificação de variáveis de interesse contidas nos dados bibliográficos ou mesmo nos textos. Por outro lado, muitos sistemas utilizados para realizar buscas e analisar informações em bases de dados bibliográficas são livres e de acesso gratuito, o que contribui para sua disseminação. Entre os muitos existentes, destacamos o Bibexcel (http://www.umu.se/inforsk/Bibexcel/) e o STANALYST (Polanco, 1995; 2008), ambos de acesso gratuito.

A análise da citação também compreende o uso intensivo de sistemas para o tratamento de informações contidas em bases de dados por intermédio da seleção de campos específicos, como Author, Co-authour e Address, esse último para identificar laboratórios e instituições, aos quais autores e coautores estão vinculados (colaborações inter e multi-institucionais). Além disso, é possível concentrar a busca em bases restritas à sistematização de dados de uma única instituição. Um exemplo é a pesquisa realizada por Beaver (1984), na base mantida pelo Williams College sobre atividades de pesquisa nas ciências naturais, psicologia e história da ciência para levantar a pesquisa colaborativa. Um importante incremento à análise de citações, sobretudo à contagem de coautores, são os modelos quantitativos e sistemas desenvolvidos para a análise de redes sociais (Carrington; Scott; Wasserman, 2007) presentes em muitos estudos sobre pesquisa científica colaborativa (Hara et al., 2003; Larsen, 2008; Sonnenwald, 2003). A partir da junção dos métodos (análise de citação, endereços e redes sociais), os pesquisadores formam redes de autores, coautores e instituições para analisar as colaborações em termos de dimensão, efeitos de produtividade e para o desenvolvimento de áreas de conhecimento ou temáticas especificas (Larsen, 2008), utilizando para tanto sistemas específicos para a visualização, de acesso gratuito como o Pajek (http://vlado.fmf.uni-li.si/pub/networks/pajek e o KrackPlot (http://www.isi.edu/ blythe/KP/) ou pagos, como o UCINET (http://www.analytictech.com/ downloaduc6.htm). O trabalho de Larsen (2008) sobre o impacto de redes de coautores na estruturação de campos de pesquisa emergentes é um exemplo da mescla da análise de citação com redes sociais.

\section{Análise de citação e a pesquisa colaborativa}

As críticas à cienciometria são fartas, e não se restringem a sua dimensão quantitativa, mas, fundamentalmente, ao reducionismo, ou seja, à redução da ciência à produção de artigos (Polanco, 1995). Aspectos privilegiados pelas abordagens sociológicas, como a formação acadêmica da equipe de pesquisa e sua constituição, o contexto sócio-político de desenvolvimento do projeto, a cultura e as relações pessoais e institucionais, além dos efeitos desses fatores na interação com diferentes áreas de pesquisa são desconsiderados (Machado; Teixeira, 2007). O contexto e sua indelével relação com o processo de produção do conteúdo em si das pesquisas científicas, princípio observado por muitas abordagens sócio-antropológicas da ciência, é desprezado pela cienciometria (Latour; Woolgar, 1986; Knorr-Cetina; 1981, 1999; Law, 1994; Machado, 2003). Como observa Polanco (1995), a abordagem cienciométrica da ciência coloca-se em uma posição diametralmente oposta aos estudos baseados na investigação de situações locais e à biografia de atores. Assim, de modo geral e definitivo, o processo de produção de conhecimentos científicos não faz parte do escopo de análise da cienciometria, bem como o processo de produção de um texto científico (Latour; Woolgar, 1986).

Por outro lado, a aproximação entre análise de coautor e a literatura especializada no estudo da pesquisa científica colaborativa não é recente. De fato, a correlação entre o aumento da relação de coautoria nos periódicos científicos e a disseminação da pesquisa científica colaborativa deve-se a Smith (1958). Os primeiros trabalhos baseados nessa correlação foram publicados na década seguinte, tendo como principal base teórica as teses de Price (Katz ; Martin, 1997; Polanco, 1995; Price; Beaver, 1966). Katz e Martin $(1995,1997)$ salientam, todavia, que Smith (1957) já ponderava que o fenômeno do crescimento da coautoria ou da múltipla autoria em um mesmo artigo não fornece uma descrição completa do tipo de relação e das atividades que redundaram no artigo.

Segundo a literatura especializada, a métrica da citação baseia-se na relação entre autor e coautor (Katz; Martin, 1997; Subramanyam, 1983; Wray, 2006; ). E o uso dessa métrica na análise da pesquisa científica colaborativa, sobretudo com relação à contabilidade da sua produtividade, parte da tese da existência de uma relação de equivalência entre as relações autor - 
coautor e de colaboração. Newman (2001) a sintetiza, ao afirmar que considera "dois cientistas conectados a uma mesma rede quando eles são coautores em um ou mais artigos" (Newman, 2001, p. 1, tradução dos autores). A (relação de) equivalência é condição de possibilidade para tomarmos a análise de citação como métrica da pesquisa científica colaborativa. Por conseguinte, entender os limites da métrica da citação para a análise da pesquisa científica colaborativa é compreender a positividade ou a negatividade da relação de equivalência. Dito de outro modo, trata-se de saber se a relação autor - coautor expressa a relação de colaboração científica entre pesquisadores de diferentes equipes, laboratórios ou instituições em um determinado momento.

primeiro passo é compreender qual tipo de relação configura a pesquisa colaborativa científica.

A pesquisa colaborativa científica não deve ser compreendida como uma soma de pesquisadores reunidos em torno de um projeto com objetivos, metas e prazos bem delineados (Wray, 2006). Não se trata, por conseguinte, de pesquisadores trabalhando paralelamente em um mesmo projeto, mas de pesquisadores trabalhando em conjunto, compartilhando problemas, objetos e, em muitos casos, insumos. Sonnenwald (2003) compreende a colaboração científica como uma forma de produção de conhecimentos técnico-científicos que suporta interações orientadas para áreas e objetivos comuns. Ela é um empreendimento que envolve o trabalho cooperativo de profissionais com diferentes qualificações e competências sociotécnicas, em diferentes laboratórios ou instituições (San Martin-Rodriguez et al., 2005). Sociotécnicas no sentido de um ator cuja ação é efeito de suas competências sócio-políticas (para agir em grupos sociais) e técnico-científicas. Ela implica, portanto, uma divisão do trabalho de pesquisa em diferentes etapas, segundo as competências e a expertise da equipe de pesquisa. Simetricamente, a colaboração interinstitucional implica que cada instituição atue segundo sua competência e expertise. Ao apoiar-se fortemente no trabalho cooperativo, a colaboração científica produz mudanças nas práticas científicas das equipes envolvidas em programas ou projetos científicos colaborativos (Katz; Martin, 1995; 1997; San MartinRodriguez et al., 2005; Wray, 2006). A organização da equipe sofre mudanças, uma vez que o processamento do material de pesquisa e/ou os experimentos já não são completamente executados pela equipe em um laboratório. Isso exige o desenvolvimento de estratégias comuns para o tratamento de dados, de comunicabilidade entre equipes e de difusão do conhecimento produzido, além da pactuação de um cronograma comum de atividades concomitante ao observado pela equipe para a execução de outros projetos externos à colaboração. Outro elemento é a perda da autonomia e independência de uma equipe sobre as estratégias experimentais. As modificações e ajustes envolvem negociações para a produção de consensos entre as equipes de pesquisa (Knorr-Cetina, 1999). Essas características da colaboração e do trabalho cooperativo são tratadas por San MartínRodríguez et al. (2005) em sua revisão sobre determinantes do sucesso da colaboração.

Assim, em principio, a pesquisa científica colaborativa produz mudanças no modo de produção do conhecimento, com impactos na divisão de trabalho, na forma como se produzem as estratégias experimentais e se realizam os experimentos. As características assumidas pela pesquisa científica colaborativa sustentam e explicam o aumento da capacidade de processar grandes volumes de dados a partir da operação de diferentes ferramentas intelectuais e técnicas. A colaboração científica implica o desenvolvimento do trabalho cooperativo, na divisão de atividades, na pactuação de atribuições entre equipes de trabalho, as quais podem ou não ser coordenados em redes ou em plataformas. Por conseguinte, há uma relação de positividade entre a relação de colaboração e o trabalho cooperativo.

Dito isso, qual tipo de implicação a relação autor - coautor conforma?

Katz e Martin $(1995 ; 1997)$ reúnem uma série de elementos para caracterizá-la e diferenciá-la da colaboração, descrevendo diferentes situações. A relação autor-coautor pode basear-se ou não em interações fortuitas e parciais entre dois ou mais pesquisadores (Katz; Martin, 1995). Katz e Martin aventam a possibilidade de dois pesquisadores efetivamente colaborarem, mas decidem, em função das estratégias de difusão de suas respectivas áreas de pesquisa, publicarem separadamente (Katz; Martin, 1995). Essa situação descrita por Katz e Martin (1995) mantém uma vinculação estreita com a política editorial de periódicos científicos de determinadas áreas de conhecimento e de pesquisa, no caso dos 
pesquisadores não pertencerem à mesma área. É possível que o pesquisador não possa (ou não tenha interesse de) publicar naquele periódico. Outro fator levado em consideração é a posição do periódico selecionado pelo autor principal na avaliação da área. O ponto sustentado por Katz e Martin (1995) é quanto à existência de algumas situações nas quais a colaboração não resulta em um artigo científico em comum. Consideramos, por nossa vez, que em todos esses casos o que está em jogo é a posição do artigo científico na estratégia de difusão do conhecimento científico de um determinado pesquisador implicado em uma colaboração.

Ainda com relação à posição do artigo, cabe ressaltar outro aspecto. $\bigcirc$ artigo científico pode não ser efeito de uma colaboração prévia; mas o ponto de partida para uma colaboração. Nesse caso, a relação ulterior não compreende o trabalho cooperativo. A colaboração começará no processo de elaboração do artigo e redundará em alguma modalidade de colaboração. É muito provável, nessa situação, que os pesquisadores em questão guardassem algum tipo de proximidade (Knoben ; Oerlemans, 2006).

Entre as relações fortuitas e parciais que redundam na publicação de um artigo científico em comum, encontramos o empréstimo de um insumo de pesquisa, considerado estratégico para a execução do trabalho de pesquisa pela equipe. Outra possibilidade, talvez mais corriqueira, é a interação por meio da expertise de um pesquisador não vinculado à equipe de pesquisa em uma determinada técnica, em um tema de pesquisa ou na interpretação de um autor. Essas situações não envolvem necessariamente a pesquisa colaborativa. Por outro lado, o pesquisador que recebeu o auxílio técnico-científico pode não caracterizá-lo e, por conseguinte, formalizá-lo, como uma prestação de serviço tecnológico ou consultoria, as quais implicariam uma remuneração. A relação não é contratual e está dissociada da esfera monetária, ou seja, do pagamento do serviço. A retribuição se consumaria na inclusão do pesquisador como coautor do artigo científico.

Outra situação ventilada por Katz e Martin (1995), é quando a citação de um pesquisador entre os coautores envolve o fortalecimento da equipe por meio da aproximação com um pesquisador altamente produtivo.

Esses mesmos autores descrevem uma série de outras situações relativas à vinculação institucional dos coautores - campo Address (Katz; Martin, 1995, p. 18, 19). Novamente a indagação é se a citação de instituições expressa, todas as vezes, uma colaboração interinstitucional ou não. Katz e Martin (1995) objetam que a colaboração pode restringir-se a um pesquisador e não envolver de fato um projeto ou programa científico colaborativo entre as duas instituições.

$\mathrm{O}$ que essas situações têm em comum? Em nenhum desses casos a relação autor - coautor é efeito da participação ativa de um ator (pesquisador ou equipe de pesquisa) na concepção da pesquisa e de todas as etapas experimentais ou trabalho de campo e, por fim, na interpretação dos resultados preliminares e finais. As situações descritas não implicaram divisão contínua de atividades técnico-científicas, na definição de um cronograma, de objetivos e de metas comuns. Isso não diminui em muitos casos a complexidade ou importância da relação para o processo de produção de conhecimentos técnico-científicos: apenas a coautoria nem sempre é efeito e expressão do trabalho cooperativo. Logo, a presença de múltiplos autores não pode ser considerada como evidência da pesquisa científica colaborativa (Katz; Martin, 1995; 1997; Wray, 2006).

Se a relação autor - coautor e a relação entre colaboradores não é equivalente em todas as situações, significa que a análise de citação não pode ser tomada como métrica da pesquisa científica colaborativa indiscriminadamente. Ela exige uma análise da situação, uma análise do que está implicado na relação autor - coautoria, muito embora possamos considerar que a análise da citação aponta para a existência de alguma modalidade de relação entre autor - coautor e/ov entre instituições naquele artigo científico. Aponta, por conseguinte, para o fato de o artigo científico ser expressão de alguma relação sociotécnica entre pesquisadores e instituições (Latour; Woolgar, 1986), porém a relação carece de qualificação. Advogamos, aqui, baseados em Katz e Martin $(1995$; 1997) que a análise da citação realizada por meio de métodos quantitativos e tomada isoladamente não permite essa qualificação, entendendo-se por qualificação a compreensão da relação, ou seja, da dinâmica da implicação entre dois ou mais pesquisadores e duas ou mais instituições. Qualificar, portanto, implica conhecer algo que os métodos quantitativos não permitem, ou seja, a relação. Qualificar implica conhecer os diversos modos de uso da relação autor coautor pelos pesquisadores em cada área do 
conhecimento (Certeau, 1996). As situações descritas por Katz e Martin (1995; 1997) indicam muitos modos de usar a relação autor - coautor. Hara et al. (2003) salientam que há diferenças no modo de se construir a relação de autor - coautor entre as áreas de conhecimento. Qualificar esses modos de usar implica, por exemplo, conhecer quais são as diferenças entre as áreas de conhecimento e estabelecer os mecanismos a partir dos quais elas se expressam na relação autorcoautor. A crítica de Subramanyam (1983) à métrica de citação para a pesquisa científica colaborativa capturou com rara sensibilidade o imperativo da qualificação. Subramanyam alerta para a limitada capacidade dos métodos de uso mais frequente dimensionarem a colaboração e auxiliarem na análise de seus impactos na organização da pesquisa científica. Mesmo porque, segundo ele, a participação e a magnitude da colaboração de um pesquisador sofrem mudanças no transcurso do desenvolvimento do projeto de pesquisa colaborativo (Subramanyam, 1983).

Não obstante os limites da análise da citação como métrica da pesquisa científica colaborativa, seu uso se mantém.

É importante considerar, ao se discutir o uso da análise de citação como métrica da pesquisa científica colaborativa, as dificuldades e a qualidade dos dados disponíveis. Senão, vejamos. Técnicas como survey e questionários têm baixa taxa de resposta e alta taxa de retorno com significativo percentual de erro na informação. Dependendo da situação analisada, a conferência da informação é difícil. A análise de citação, por seu turno, utiliza informações disponíveis em bases de dados, as quais independem do preenchimento individual. $\bigcirc$ autor principal é responsável pelos dados informados - nome, titulação, filiação institucional -e relativos a todos os autores. A amplitude das colaborações entre equipes de pesquisa de diferentes laboratórios (teamwork), entre áreas (multidisciplinar), instituições (multi-institucional) e países (Beaver; 2001 ; Chompalov; Shrum, 1999; Cummings; Kiesler, 2005; Larsen; 2008) dificulta a obtenção de informações mais precisas e, sobretudo, abertas a consulta pública, justificando em parte a disseminação da análise da citação.

À acessibilidade dos dados juntam-se outras características da análise da citação que corroboram sua disseminação. Entre os sistemas de tecnologia da informação utilizados, há uma diversidade de acesso gratuito. Nesse caso, os custos para elaboração da pesquisa nas bases de dados diminuem. As ferramentas (de acesso gratuito ou não) suportam a busca em um número ampliado de campos, aumentando o volume de dados para análise. Assim, um ponto importante para compreendermos a disseminação duradoura da análise de citação é a potência do volume numérico de informação (artigos $X$ período de tempo Xárea de conhecimento) que os sistemas permitem gerar.

\section{À Guisa de Conclusão}

Partimos de quatro (4) questões, a saber: 1) até que ponto a métrica da análise de citação permite dimensionar os efeitos das colaborações científicas na produção de conhecimentos científicos; 2) a análise de citações é uma medida do conhecimento técnicocientífico produzido por uma colaboração científica; 3) a presença de múltiplos autores caracteriza de fato uma pesquisa científica desenvolvida no âmbito da colaboração entre equipes de diferentes laboratórios ou instituições; 4) se essa métrica permite avaliar o impacto dos programas colaborativos na produção de conhecimentos técnico-científicos com potencial inovador para os diferentes setores da economia. E, ao longo da argumentação, procuramos demonstrar que a análise da citação não é uma métrica absoluta da pesquisa científica colaborativa. Primeiro, ela não permite saber se o artigo foi concebido no âmbito de uma relação colaborativa. Segundo, não permite dimensionar efeitos das colaborações científicas na produção de conhecimentos científicos. A pesquisa colaborativa é uma relação diversa da relação autor coautor, embora em algumas situações de fato haja equivalência. E nessas situações especificas (e apenas nelas), o artigo científico expressa as duas relações colaboração científica e autoria. Para empreendermos uma análise mais acurada dessas relações, é preciso compreender a(s) posição(ões) dos artigos científicos na colaboração, bem como na estratégia de difusão de resultados seguidas pelos atores (pesquisadores e instituições), mediante a avaliação de produtividade das diferentes áreas de conhecimento (Hara et al., 2003; Velho, 1999).

Logo, a métrica baseada na análise de citação não é uma medida completa do conhecimento técnicocientífico produzido no âmbito de uma colaboração científica. A presença de múltiplos autores, do mesmo 
modo, não pode ser tomada de imediato, sem uma análise mais acurada das relações que motivaram a citação de autores, como expressão de uma colaboração científica. Com relação à métrica do potencial inovador de conhecimentos científicos para os diferentes setores da economia, a análise de citação pode apontar casos que deverão ser objeto de estudos específicos.

Quando deslocamos a questão da métrica da citação e sua relação com a pesquisa científica colaborativa para espaços de produção de conhecimento específicos, os problemas e limites aqui levantados adquirem complexidade. No caso das iniciativas de pesquisa colaborativa realizadas por instituições latino-americanas e caribenhas, outros questionamentos se somam. A literatura especializada é unívoca ao salientar que há pouco conhecimento sistematizado "sobre a natureza, o caráter, o funcionamento e a organização da pesquisa científica em países cientificamente periféricos" (Velho, 1999, p. 2). Nesse sentido, Frame (1985) chama a atenção para o fato de desconhecermos se o comportamento de citações nesses países segue os padrões apontados pela literatura especializada para outros espaços científicos mais estudados, como as comunidades acadêmicas norte-americanas e de países da Europa ocidental. Em relação às colaborações científicas, há poucas análises concluídas (Teixeira et al., 2008b) que nos permitam inferir como as publicações são usadas, se há predomínio de publicações internas ou externas e quais estratégias orientam a relação autor - coautor.

Hara et al. (2003) argumentam que há diferenças entre as áreas de conhecimento no tocante à colaboração. Sua natureza deve ser aprofundada, para 0 desenvolvimento de métricas mais adequadas a pesquisa científica colaborativa. Essas diferenças, que em alguns casos se manifestam no lugar do artigo no processo de produção do conhecimento, e a maior ou menor tradição de desenvolver o projeto por meio do trabalho cooperativo também deverão ser contempladas na análise.

Porém, ainda assim, a métrica da citação não deve ser de todo desconsiderada para o desenho de políticas de avaliação da pesquisa científica colaborativa. Além da acessibilidade aos dados, da disponibilidade de ferramentas e o custo/tempo para realização de pesquisas, é importante considerar que essa modalidade de análise aponta para a existência de relações. Ela contribui para a seleção de situações que poderão ser alvo de estudos mais aprofundados.

Por fim, a observação de Subramanyam (1983) acerca das mudanças na magnitude da colaboração de um pesquisador ao longo do desenvolvimento do projeto colaborativo aponta as dificuldades para empreendermos uma análise da pesquisa científica colaborativa em bases qualitativas. Análises mais precisas, sobretudo para o desenho de políticas de pesquisa e sua avaliação, precisarão mesclar técnicas quantitativas e qualitativas.

\section{REFERÊNCIAS}

BEAVER, D.B. Refletions on scientific collaboration (and its study): past, present and future. Scientometrics, v.52, n.3, p. 365-377, 2001.

BEAVER, D. de B.: a step beyond collaboration. George Sarton centennial, Communication and Cognition, Ghent, Belgium: p.449-452.1984. Disponível em:<http://www.akademiai.com/ index/FA5LNKLHDKGD7X77.pdf>. Acesso em: 29 ago. 2009.

BOZEMAN, B, BOARDMAN, C. Research and technology collaboration and linkages: implications from two U.S. case studies. In: REPORT to Council of Science and Technology Advisors (CSTA) Study on Federal S\&T Linkages. 2003.

CARRINGTON, P., SCOTT, J., WASSERMAN, S. Models and methods in social network analysis. New York: Cambridge University Press, 2007.

CERTEAU, Michel A invenção do cotidiano. Rio de Janeiro: Vozes, 1996.
CHOMPALOV, I.; SHRUM, W. Institutional collaboration in science: a typology of technological practice. Science, Technology, \& Human Values, v. 24, n.3, p. 338, 372, 1999.

CUMMINGS, J.; KIESLER, S. Collaborative research across disciplinary and organizational boundaries. Social Studies of Science, v.35, n.5, p.703-722, 2005.

D'AMOUR, D. et al. The conceptual basis for interprofessional collaboration: core concepts and theoretical frameworks. Journal of Interprofessional Care, suppl. 1, p. 1 16-131, 2005.

FRAME, J. D. Problems in the use of literature-based S\&T indicators in developing countries In: MORITA-LOU, H. (org). Science and technology indicators for development. Boulder: Westview Press, 1985. p.117-122.

GODIN, B. A cultura dos números: as origens e o desenvolvimento da estatística na ciência. RECIIS, v.2, n. 1, p. 7-18, 2008. 
GODIN, B. La science sous observation: cent ans de mesure sur les scientifiques 1906 - 2006. Québec: Ed. Les Presses de L’Université Laval, 2005. 81 p.

HARA, N. et al. An emerging view of scientific collaboration: scientists perspectives on collaboration and factors that impact collaboration. Journal of the American Society for Information Science and Technology, v.54, n. 10, p. 952-965, 2003.

KATZ, J. S.; MARTIN B. What is research collaboration? Research Policy, v.26, p. 1-8, 1997.

KATZ, J.S.; MARTIN B. What is research collaboration? Report. Science Policy and Research Evaluation Group. ESRC Centre for Science, Technology, Energy and Environment Policy. Science Policy Research Unit. University of Sussex. Falmer, Brighton. BN1 9RF, UK. 1995. Disponível em: <http://www.sussex.ac.uk/Users/ sylvank/pubs/Res col9.pdf>. Acesso em: 29 ago. 2009.

KNOBEN J.; OERLEMANS, L.A.G. Proximity and interorganizational collaboration: a literature review. International Journal of Management Reviews, v.8, n.2, p. 71-89, 2006.

KNORR-CETINA, K. Epistemic cultures: how the sciences make knowledge. Cambridge: Harvard University Press, 1999.

KNORR-CETINA, K. The manufacture of knowledge: an essay on the constructivist and contextual nature of science. Oxford : Pergamon, 1981.

LARSEN, K. Knowledge network hubs and measures of research impact, science structure and publication output in nanostructured solar cell research. Scientometrics, v.74, n. 1, p. 123-142, 2008.

LATOUR, B.;WOOLGAR, S. Laboratory life: the construction of scientific facts. 2. ed. Princeton : Princeton University Press, 1986.

LAW, J. Organizing modernity. Oxford : Blackwell, 1994.

LEYDESDORFF, L. The challenge of scientometrics: the development, measurement and self-organization of scientific communications. [S.I.]: Universal Publishers, 2001

LONGO W. P.; OLIVEIRA A. R. P. Pesquisa cooperativa e centros de excelência. Parcerias Estratégicas, v.9, p. 129-144, 2000.

MACHADO, C.J.S. ; TEIXEIRA M.O. Descoberta, invenção e inovação segundo os estudos sociais anglo-saxões e europeus das ciências. DataGramaZero, v. 8, n.2, 2007 Disponível em: http:// unw.dgz.org.br/abr07/Art 03.htm. 2007. Acesso em: 27 ago. 2009.

MACHADO, C.J.S. Tecnologia, meio ambiente e sociedade: uma introdução aos modelos teóricos. Rio de Janeiro: e-Papers, 2003.

MORITA-LOU,H. (Org.) Science and technology indicators for development. Boulder: Westview Press, 1985.

NEWMAN, M.E.J. Scientific collaboration networks I: network construction and fundamental results. Physical Review, v.64, n. 1, p. $1-8,2001$

OCDE. The measurement of scientific and technical activities: proposed standard practice for survey of research experimental development. 1962. DAS/PD/62.47.
POLANCO, X. Aux sources de la scientométrie: dossier Solaris, n.2: Les sciences de l'information: bibliométrie, scientométrie, infométrie. Solaris, n. 2, 1995. Disponível em: <http:// www.info.unicaen.fr/bnum/jelec/Solaris/d02/2polanco 1.html. 1995 >. Acesso em: 27 ago. 2009.

POLANCO, X. Stanalyst: uma aplicación para nuevos estúdios bibliométricos sobre bases de datos locales. In: SEMINARIO INTERNACIONAL SOBRE INDICADORES DE CIENCIA, TECNOLOGÍA E INNOVACIÓN, 2006, Santiago. Anais. 2006.

POLANCO, X. Transformer l'information en connaissance avec Stanalyst: cadre conceptuel et modele. Encontros Bibli: Revista Eletrônica de Biblioteconomia e Ciência da Informação, n. Esp., 2008. Disponível em: <http://www.periodicos.ufsc.br/index.php/ eb/article/view/1 122>. Acesso em: 27 ago. 2009.

PRICE, D. J. de S.; BEAVER, D. de B. Collaboration in an invisible colleges. American Phychologist, v. 21, p.1011-1018, 1966.

SAN MARTIN-RODRIGUEZ, $L$. et al. The determinants of successful collaboration: a review of theoretical and empirical studies. Journal of Interprofessional Care, suppl. 1, p. 132-147, 2005.

SHRUM, W.; GENUTH, J.; CHOMPALOV, I. Structures of scientific collaboration. Cambridge: The MIT Press, 2007.

SMITH, M. The trend toward multiple authorship in Phychology. American Psychologist, v. 13, p. 596-599, 1958.

SONNENWALD, D. The conceptual organization: an emergent organizational form for collaborative R\&D. Science and Public Policy, v.30, n.4, p.261-272, 2003.

SUBRAMANYAM, K. Bibliometric studies of research collaboration: a review. Journal of Information Science, v.6, n. 1, p.33-38, 1983.

TEIXEIRA, M. O. et al. Análise da utilização de redes cooperativas como dispositivo de uma política de desenvolvimento tecnológico de insumos em saúde: o caso do PDTIS / Fiocruz. In: JORNADAS LATINOAMERICANAS DE ESTUDIOS SOCIALES DE LA CIENCIA Y LA TECNOLOGIA - ESOCITE, 7., 2008, Rio de Janeiro. Anais... Rio de Janeiro: 2008a.

TEIXEIRA, M. O. et al. Descrição e análise do uso de redes cooperativas como instrumento de coordenação da pesquisa científica em saúde. Revista Políticas Públicas, 2009a. No prelo.

TEIXEIRA, M. O. et al. Pesquisa colaborativa em saúde: considerações sobre o uso de redes cooperativas na pesquisa tecnológica em biomedicina. Revista Ciência e Saúde Coletiva, 2009b. No prelo. Disponível em: <http:// www.abrasco.org.br/cienciaesaudecoletiva/artigos/ artigo_int.php?id_artigo $=328>$.

TEIXEIRA, M. O. La science sous observation: cent ans de mesure sur lês scientifiques 1906 - 2006. Revista Eletrônica de Comunicação, Informação e Inovação em Saúde, v.2, n. 1, 2008b. Disponível em: <http://www.reciis.cict.fiocruz.br/index.php/reciis/ article/view/161/155>. Acesso em: 27 ago. 2009. 
TEIXEIRA, M. O. et al. Science, technology and health innovation in Brazil: an experience of re-organization of scientific work in a public research institution in biomedicine. In: CONGRESS SOCIETY FOR SOCIAL STUDIES OF SCIENCE \& EUROPEAN ASSOCIATION FOR THE STUDY OF SCIENCE AND TECHNOLOGY (EASST), 4. 2008, Rotterdam. Proceedings... Roterdam, 2008c.

TEIXEIRA, M. O. et al. O uso de ferramentas de TI e a pesquisa colaborativa em saúde: análise de uma experiência local. [S.I.]: [s.n.], 2009c. Mimeografado.

VELHO, L. Como estabelecer um sistema de indicadores bibliométricos para a América Latina. [S.I.]: [s.n.], 1999.
Mimeografado. Disponível em: <http://www.redhucyt.oas.org/ ricyt/interior/biblioteca/2brvelho.PDF.> . Acesso em 27 ago. 2009.

WRAY, K. B. Scientific authorship in the age of collaborative research. Studies in History and Philosophy of Science, v.37, p. 505-514, 2006.

Agradecemos ao Conselho Nacional de Desenvolvimento Científico e Tecnológico (CNPq) e a Fundação Carlos Chagas de Amparo à Pesquisa do Estado do Rio de Janeiro (FAPERJ) o apoio para a realização da pesquisa e elaboração desse artigo. 